\title{
Towards the use of Policy Decomposition for Composite Service Management by Non-expert End-users
}

\author{
John Keeney, David Lewis, Vincent Wade \\ Knowledge \& Data Engineering Group (KDEG) \& FAME, \\ School of Computer Science \& Statistics, Trinity College Dublin, Dublin, Ireland. \\ \{John.Keeney,Dave.Lewis, Vincent.Wade\}@cs.tcd.ie
}

With the advent of Web 2.0, as web service composition gets easier, there is a trend towards nonexpert users not just consuming information and services, but also providing, aggregating, composing and eventually managing their own information, services and resources. However non-expert users need to manage their composed services in a way they can understand. Policy-based management promises the ability to control services in a consistent manner via high-level declarative directives, constraints and goals. However, a significant drawback of policy-based management for complex systems remains the lack of an automated mechanism to resolve the meaning of high-level goals so they can be enforced $[1,2]$.

We propose an approach to compose the available heterogeneous management interfaces of underlying constituent services to produce a coherent higher-level management interface for the composite service. This composed management interface can then be presented to the user at a level of abstraction that corresponds to non-expert user's needs thereby allowing the user to express their specific requirements. Hence the user can manage their composite service, in a manner tailored to them, without the burden of needing to understand how to manage each constituent service. This supports lower-cost user-initiated management, without access to IT experts, thereby increasing productivity and lowering operating costs.

To motivate this research, consider a customer support department in a small enterprise where a customer services manager is in charge of a business process to track customer requests and follow-on actions. The business process is supported by a service-oriented platform composed from a set of constituent services including: a trouble-ticket tracking service, a knowledge base of support articles, a messaging/paging service, a billing service, etc.. It is unlikely that the manager personally composed these services together to build this platform, however, in a small enterprise it is likely that the manager is expected to manage, adapt and personalise such a platform in an ongoing manner.

The manager is likely to be more concerned with macro-level business issues than low-level interfaces.
The manager must ensure that high-priority requests are handled quickly; that valued customers get higher priorities and agreed service levels; that new service levels, tariffs and contracts can be easily merged with others in a manner that maintains fairness; etc..

Advanced human-led configuration and operation management requires the abstraction of complex tasks for the human to understand how to iteratively view, control and compose these tasks at a higher-level. In order to make sense of a complex management task, a user must be able to understand the information and mechanism of the task and be able to abstract and contextualise information and mechanisms into their own personal viewpoint. However this abstraction process must be performed in a lossless and reversible manner so that high-level management tasks and goals can be mapped back to low-level actions.

For example, the approach taken in [3] focuses on identifying a user's policies and goals to build a service orchestration (process) that can match those goals. However, when non-expert users compose services to achieve a task their objective is a system that provides their application-level requirements and provides some value. The value or benefit that a user gets from a service in a composition depends on the requirements of the user and how the service interacts with the other services in the composition.

There is a small body of work on management policy composition/de-composition targeting policy refinement, the mapping of high-level goals to lowlevel enforceable policies. A conclusion that can be drawn from this work is that the single direction refinement of abstract goals to enforceable policies is extremely difficult $[1,2]$. This refinement step must be preceded by an examination and composition of possible underlying policies to produce a large search/state space (e.g. [4]); AI-based search or training techniques to identify partial goal satisfaction combinations (e.g. $[2,4,5,6])$; or a set of composed high-level policy templates (e.g. [7,8]).

Several policy languages exploit ontological semantics to arrange management concepts taxonomically, allowing abstract concepts (higher up) to be used in place of deeper, concrete concepts, so 
policies can be represented at different levels of abstractions [10], or as a policy-continuum [9], from high-level policies to low-level enforceable policies. The use of semantics for policy refinement is also described in [10] where higher level descriptions of management actions are modelled in an upper ontology and mapped (with the help of rules) to implementationspecific ontologies for management interfaces. In some cases the ontologies are automatically derived from non-semantic management interfaces to interoperate between different types of interfaces $[10,11]$.

We propose an approach that exploits ontological semantics to describe management state, actions and policies of the constituent services that make up a service composition. These can then be combined with a rich model of the orchestration of services to make up the composite service (already provided as part of the composition process). When also combined with a semantic model of the manager's competencies, goals, and values then these components can be presented at an appropriate level of abstraction.

Based on these semantic descriptions of the constituent services, the capabilities of the user, and a model of the composition, the available low-level management capabilities of the constituent services can be iteratively combined and abstracted towards a management interface that fits the users capabilities. This semantically enhanced bottom-up approach supports capturing the user's requirements specified according to the high-level abstracted capabilities of the composite service. This approach then natively supports mapping these high-level requirements / goals to the underlying capabilities of the individual constituent services, guided by the semantic mark up of these capabilities.

Rather than make assumptions about the manager's goals, and have the policies composed automatically, a high-level toolkit is required for the non-expert manager to define and compose policies at a level of abstraction appropriate to them. This approach does not follow the established goal refinement approach, where low-level management policies and actions are expressed as low-level goals $[1,4,12]$ and only then automatically composed and decomposed to/from high-level. Rather than convert low-level capabilities to goals, or acceptable ranges [13], at a low level, the mapping to/from management goals is performed after the constituent services have been composed, thereby exploiting more semantics from the manager and from the composition process. This addresses the problem where the management assumptions that appeared to make sense at a lower level when dealing with individual services in isolation, but instead frustrate the manager when the service is composed because higher level requirements and considerations now need to be addressed, thereby taking a holistic view of the managed composite system rather than composing manageable constituent elements.

\section{Acknowledgement}

Work is funded by the Irish Government as part of the FAME SFI Strategic Research Cluster (Grant No 08/SRC/I1403)

\section{References}

[1] Bandara, A., Lupu, E., Moffett, J., Russo, A., "A Goalbased Approach to Policy Refinement", Policies for Distributed Systems and Networks (POLICY), 7-9 June 2004

[2] Beigi, M., Calo, S., Verma, D., "Policy Transformation Techniques in Policy-based Systems Management", Policies for Distributed Systems and Networks (POLICY), 7-9 June 2004

[3] Kaiser, M., "Toward the Realization of Policy-Oriented Enterprise Management”. Computer, 40(11), November 2007

[4] Rubio-Loyola, J., Serrat, J., Charalambides, M., Flegkas, P., Pavlou, G., Lafuente, A.L., "Using linear temporal model checking for goal-oriented policy refinement frameworks", Policies for Distributed Systems and Networks (POLICY), 68 June 2005

[5] Keeney, J., Wade, V., "Towards Policy Decomposition for Autonomic Systems Governance by Applying Biologically Inspired Techniques", International IEEE Workshop on Managing Ubiquitous Communications and Services (MUCS), Salvador, Bahia, Brazil, 11 April 2008

[6] Jennings, B., van der Meer, S., Balasubramaniam, S., Botvich, D., O'Foghlu, M., Donnelly, W., Strassner, J., "Towards Autonomic Management of Communications Networks", IEEE Communications, 45(10), October 2007

[7] Liao, B., Gao, J., "An Automatic Policy Refinement Mechanism for Policy-Driven Grid Service Systems" Conference on Grid and Cooperative Computing (GCC), Beijing, China, 30 November-3 December 2005

[8] Casassa Mont, M., Baldwin, A., Goh, C, "POWER prototype: towards integrated policy-based management", Network Operations and Management Symposium (NOMS), Hawaii, 10-14 April 2000

[9] Strassner, J, "Policy-Based Network Management Solutions for the Next Generation”, Morgan Kauffman, 2003

[10] Guerrero, A., Villagrá, V., López de Vergara, J., Sánchez-Macián, A., Berrocal, J., "Ontology-based Policy Refinement Using SWRL Rules for Management Information Definitions in OWL", Distributed Systems: Operations and Management (DSOM), 23-25 October 2006

[11] Lehtihet, E., Agoulmine, N., "Towards integrating management interfaces". Network Operations and Management Symposium (NOMS), 7-11 April 2008

[12] Darimont R., van Lamsweerde, A., "Formal Refinement Patterns for Goal-Driven Requirements Elaboration", Foundations of Software Engineering (FSE4), 1996

[13] Udupi Y.B., Sahai A., Singhal S., "A ClassificationBased Approach to Policy Refinement", Integrated Network Management (IM), Munich, Germany, 21-25 May 2007 